\title{
Coulomb Blockade Oscillations in the Thermopower of Open Quantum Dots.
}

\author{
A.V. Andreev ${ }^{1}$ and K.A. Matveev ${ }^{2}$ \\ ${ }^{1}$ Department of Physics, University of Colorado, CB 390, Boulder, CO 80309 \\ ${ }^{2}$ Department of Physics, Duke University, Durham, NC 27708-0305
}

(Draft: November 4, 2018)

\begin{abstract}
We consider Coulomb blockade oscillations of thermoelectric coefficients of a single electron transistor based on a quantum dot strongly coupled to one of the leads. Analytic expression for the thermopower as a function of temperature $T$ and the reflection amplitude $r$ in the quantum point contact is obtained. Two regimes can be identified: $T \ll E_{C}|r|^{2}$ and $T \gg E_{C}|r|^{2}$, where $E_{C}$ is the charging energy of the dot. The former regime is characterized by weak logarithmic dependence of the thermopower on the reflection coefficient, in the latter the thermopower is linear in the reflection coefficient $|r|^{2}$ but depends on temperature only logarithmically.
\end{abstract}

PACS numbers: 73.23.Hk, 73.50.Lw

Thermoelectric effects in mesoscopic devices have been the subject of extensive experimental and theoretical research [1] 8]. The particle-hole asymmetry required for such effects can be strongly enhanced in these systems as compared to the bulk materials. This and the small size of such devices make them promising candidates for technological applications, such as micro-refrigerators [3]. In particular, many experimental and theoretical studies in the last few years have focused on the thermoelectric properties of quantum dots in the Coulomb blockade (CB) regime [4]. Most of them concentrated on the $\mathrm{CB}$ oscillations of the thermopower, $S=-\frac{\Delta V}{\Delta T}$, where $\Delta T$ is the temperature difference across the dot, and $\Delta V$ is the voltage necessary to nullify the current.

The theory of the CB oscillations in the thermopower of quantum dots in the weak tunneling regime was constructed in Ref. [4]. This theory takes into account only the lowest order tunneling processes, i.e. the sequential tunneling, and neglects the cotunneling processes. Its results were in agreement with the experiments of Ref. [5]. Later [6] it became possible to experimentally access the regime of lower temperatures and stronger tunneling where the cotunneling processes become dominant. The theoretical description of this regime was recently given in Ref. [7].

In very interesting recent experiment 8] the $\mathrm{CB}$ oscillations in the thermopower of a nearly open quantum dot were studied as a function of the reflection coefficient $|r|^{2}$ in the contact. The setup of these experiments is schematically represented in Fig. 1. Surprisingly, an initial decrease in the amplitude of CB oscillations of thermopower with decreasing $|r|^{2}$ was followed by a plateau with nearly $|r|$-independent CB oscillations of thermopower. This saturation was attributed [8] to the effects of elastic cotunneling [9].

The theory of thermopower for the weak tunneling regime developed in Refs. [4.:7] does not apply to this case. An additional motivation for studying the thermoelectric phenomena in such devices arises from the fact that due to the increased transparency of the contacts, the open dots are better candidates for micro-refrigerator devices [3] than the closed ones.

In this Letter we present a theory of thermoelectric effects in a quantum dot in the nearly open regime. We consider a quantum dot which is coupled by a tunneling junction to the left lead and by a single channel quantum point contact (QPC) to the right lead, see Fig. 1. The reflection amplitude in the QPC is assumed to be small, $|r| \ll 1$. The mean level spacing $\delta$ in the dot is assumed to be vanishingly small. This is a good assumption since experimentally [8] $\delta \ll T$.

The previous studies of such systems were devoted to their thermodynamic and transport properties [10 12]. A special feature of the thermoelectric power $S$ is that it is sensitive to the average energy transported by electrons, which in the tunneling approximation depends on the odd part of the density of states (DoS) as a function of energy. Thus the thermoelectric phenomena represent an independent probe of these systems.

In this Letter we find the thermoelectric coefficient $G_{T}$ of the device in Fig. 11 describing the current response $I$ at zero bias, $\Delta V=0$, to the difference of the temperatures $\Delta T$ between the two leads: $G_{T}=\left.\lim \frac{I}{\Delta T}\right|_{\Delta V=0, \Delta T \rightarrow 0}$. Our main result is the following expression for $G_{T}$ of the dot:

$$
\begin{aligned}
G_{T}= & \frac{G_{L}|r|^{2} T}{6 \pi e E_{C}} \ln \frac{E_{C}}{T+\Gamma} \sin (2 \pi N) \\
& \times \int_{-\infty}^{+\infty} \frac{x^{2}\left(x^{2}+\pi^{2}\right)}{\left[x^{2}+(\Gamma / T)^{2}\right] \cosh ^{2}(x / 2)} d x .
\end{aligned}
$$

Here $G_{L} \ll e^{2} / h$ is the conductance of the left contact, $e$ is the absolute value of the electron charge, $E_{C}$ is the charging energy. We have also introduced the energy scale $\Gamma=\left(8 \gamma / \pi^{2}\right) E_{C}|r|^{2} \cos ^{2}(\pi N)$, which depends on the gate voltage $N$; here $\ln \gamma=\mathbf{C} \approx 0.5772 \ldots$ is the Euler constant. The result (17) was obtained with logarithmic accuracy assuming that $E_{C} \gg T, \Gamma$. 


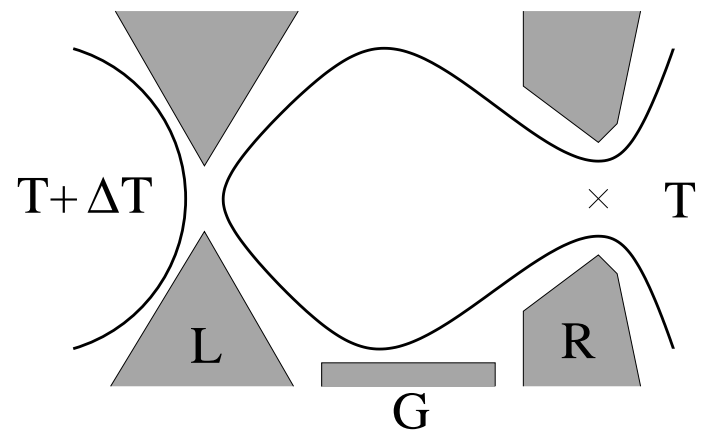

FIG. 1. Schematic drawing of a quantum dot electrostatically defined on a surface of a two-dimensional electron gas. The dot is connected to the left lead with temperature $T+\Delta T$ by a tunneling junction and to the right lead at temperature $T$ by a single channel quantum point contact. The latter is characterized by a reflection amplitude $r$ in the contact and can be thought of as arising from a point scatterer depicted by a cross. The value of $r$ is controlled by the voltage on gate $\mathrm{R}$. The voltage on the central gate $\mathrm{G}$ determines the optimal electron number $N$ in the dot.

The thermopower $S=G_{T} / G$ is then obtained from Eq. (1) using the result of Ref. [12] for the conductance $G$ of the device shown in Fig. 1, which we reproduce here for completeness:

$$
G=\frac{G_{L} \Gamma}{8 \gamma E_{C}} \int_{-\infty}^{+\infty} \frac{x^{2}+\pi^{2}}{\left[x^{2}+(\Gamma / T)^{2}\right] \cosh ^{2}(x / 2)} d x .
$$

For the two limiting cases, $T \ll \Gamma$ and $T \gg \Gamma$, we obtain simplified expressions for the thermopower:

$$
S= \begin{cases}\frac{64 \gamma|r|^{2}}{9 \pi^{2} e} \ln \frac{E_{C}}{T} \sin (2 \pi N), & \text { for } T \gg \Gamma, \\ \frac{\pi^{3} T}{5 e E_{C}} \ln \frac{E_{C}}{\Gamma} \tan (\pi N), & \text { for } T \ll \Gamma .\end{cases}
$$

It is difficult to make a direct comparison of the results (11), (3) with the experiments of Ref. [8] since the experimental data were presented in terms of a fit to the weaktunneling theory of Ref. [4. Nevertheless we want to point out that even without taking into account the elastic cotunneling effects [9], in the regime $T \ll \Gamma$ the thermopower only weakly (logarithmically) depends on the reflection coefficient, which is consistent with the observation in Ref. [8] of the thermopower virtually independent of the reflection coefficient. In the opposite regime, $\Gamma \ll T$, the thermopower is nearly independent of the temperature, but scales linearly with the reflection coefficient $|r|^{2}$ vanishing, as expected, at perfect transmission. Note that even at very low temperatures $T \ll E_{C}|r|^{2}$, one still has $T \gg \Gamma$ near half-integer values of the gate voltage $N$ corresponding to the CB peaks of conductance (2). The width $\delta N$ of those regions can be easily found from the condition $\Gamma(N) \sim T$. Upon substitution into Eq. (3) it gives the estimate of the amplitude of the CB oscillations of the thermopower $S_{0} \sim e^{-1}|r| \sqrt{T / E_{C}} \ln \left(E_{C} / T\right)$.

It is interesting to point out that in the low temperature regime $T \ll \Gamma$, when the conductance (2) shows
12] the temperature dependence $G \propto T^{2}$ characteristic of inelastic cotunneling [13], the thermopower can be expressed in terms of the logarithmic derivative of the conductance with respect to the gate voltage $2 E_{C} N$ :

$$
S=\frac{\pi^{2} T}{10 e E_{C}} \ln \left(\frac{E_{C}}{\Gamma}\right) \frac{\partial \ln G}{\partial N} .
$$

This form is analogous to the Cutler-Mott formula 14 for the thermopower of a system of non-interacting electrons in a metal, but with a different coefficient in front of the logarithmic derivative. A similar Cutler-Mott type relation holds in the case of weak inelastic cotunneling [7]; however, the prefactor of Eq. (4) contains an additional large logarithmic factor $\ln \left(E_{C} / \Gamma\right)$. In the opposite case of high temperature $T \gg \Gamma$ no expression similar to the Cutler-Mott formula applies.

Below we present the derivation of the result (1). Following Ref. 12, the electron transport through the right QPC can be described by a one-dimensional model amenable to bosonization, whereas the left contact can be treated in the tunneling approximation. The Hamiltonian of the dot has the form $\hat{H}=\hat{H}_{0}+\hat{H}_{R}+\hat{H}_{L}+\hat{H}_{C}$, where

$$
\begin{aligned}
\hat{H}_{0} & =\sum_{k \alpha} \epsilon_{k} a_{k \alpha}^{\dagger} a_{k \alpha}+\sum_{p \alpha} \epsilon_{p} a_{p \alpha}^{\dagger} a_{p \alpha} \\
& +\frac{v_{F}}{2 \pi} \sum_{\alpha} \int\left\{\left[\nabla \phi_{\alpha}(x)\right]^{2}+\pi^{2} \Pi_{\alpha}^{2}(x)\right\} d x \\
\hat{H}_{L} & =\sum_{k p \alpha}\left(v_{t} a_{k \alpha}^{\dagger} a_{p \alpha} F+v_{t}^{*} a_{p \alpha}^{\dagger} a_{k \alpha} F^{\dagger}\right), \\
\hat{H}_{R} & =\frac{D}{\pi}|r| \sum_{\alpha} \cos \left[2 \phi_{\alpha}(0)\right], \\
\hat{H}_{C} & =E_{C}\left[\hat{n}+\frac{1}{\pi} \sum_{\alpha} \phi_{\alpha}(0)-N\right]^{2} .
\end{aligned}
$$

The operators $\hat{H}_{R}, \hat{H}_{L}$, and $\hat{H}_{C}$ describe the backscattering in the right QPC, tunneling through the left contact, and the charging energy of the dot, respectively. In the equations above $\alpha=\uparrow, \downarrow$ is the spin label, $a_{p \alpha}$ and $a_{k \alpha}$ are electron annihilation operators in the dot and the left lead respectively, $D$ is the energy cutoff in the bosonization, and $\phi_{\alpha}$ is the bosonization displacement operator describing the electron transport through the right QPC with $\Pi_{\alpha}$ being its conjugate momentum, $\left[\phi_{\alpha}(x), \Pi_{\alpha^{\prime}}\left(x^{\prime}\right)\right]=i \delta\left(x-x^{\prime}\right) \delta_{\alpha, \alpha^{\prime}}$ (we have put $\hbar=1)$. The modified form of the tunneling Hamiltonian in Eq. (5b) reflects the fact that the electron tunneling event changes the electron number $\hat{n}$ in the dot. This is achieved through the introduction of the chargelowering operator $F$ [12] which satisfies the commutation relation $[F, \hat{n}]=F$. The current operator through the left contact can be obtained from the equation of motion for the charge operator $\hat{I}=-e \dot{\hat{n}}=i e[\hat{n}, \hat{H}]$. Only 
$\hat{H}_{L}$ contributes to this commutator and gives rise to the following expression for the current operator

$$
\hat{I}=i e \sum_{k p \alpha}\left(v_{t}^{*} a_{p \alpha}^{\dagger} a_{k \alpha} F^{\dagger}-v_{t} a_{k \alpha}^{\dagger} a_{p \alpha} F\right) .
$$

We treat the problem in the lowest order in the tunneling Hamiltonian Eq. (5b). We also assume that the conductance of the tunneling contact is much less than the conductance quantum, $G_{L} \ll e^{2} / h$. In this approximation all of the temperature drop happens across the left contact. We take the temperature of the left lead to be $T+\Delta T$ and that of the dot and the right reservoir to be $T$. In the linear approximation in $\Delta T$ the current $I$ can be expressed through the tunneling $\operatorname{DoS} \nu(\epsilon)$ in the dot as,

$$
G_{T}=\frac{I}{\Delta T}=\frac{G_{L}}{4 T^{2} e \nu_{0}} \int_{-\infty}^{\infty} \frac{\nu(\epsilon) \epsilon d \epsilon}{\cosh ^{2}\left(\frac{\beta \epsilon}{2}\right)} .
$$

Here $\nu_{0}$ is the DoS in the dot in the absence of interaction, Eq. (5d).

Thus, technically the problem in the tunneling approximation reduces to the calculation of the energydependent tunneling DoS, $\nu(\epsilon)$. We note that $G_{T}$ depends only on the odd (as a function of energy) component of DoS, whereas the conductance $G$ depends only on the even one. Therefore, as was mentioned earlier, thermopower measurements represent an independent test of the theory of Coulomb blockade in nearly open dots developed in Refs. 10 12]. Moreover, in the leading order in $\max \{T, \Gamma\} / E_{C}$ the odd component of the tunneling DoS vanishes 12. The thermoelectric coefficient $G_{T}$ is small in the the ratio of $\max \{T, \Gamma\} / E_{C}$ in comparison to the conductance $G$. Its calculation requires going beyond the previously adopted approximations [12] and retaining sub-leading order in $\epsilon / E_{C}$ in the tunneling DoS, $\nu(\epsilon)$.

The tunneling DoS in the dot can be expressed as

$$
\nu(\epsilon)=-\frac{1}{\pi} \cosh \frac{\beta \epsilon}{2} \int_{-\infty}^{\infty} \mathcal{G}\left(\frac{\beta}{2}+i t\right) \exp (i \epsilon t) d t,
$$

where $\mathcal{G}\left(\frac{\beta}{2}+i t\right)$ is the Matsubara Green function,

$$
\mathcal{G}(\tau)=-\sum_{p p^{\prime}}\left\langle T_{\tau} a_{p \alpha}(\tau) F(\tau) a_{p^{\prime} \alpha}^{\dagger}(0) F^{\dagger}(0)\right\rangle,
$$

analytically continued to complex time $\tau=\frac{\beta}{2}+i t$. The angular brackets $\langle\ldots\rangle$ in Eq. (9) denote the thermal average.

Because the dynamics of the operators $a_{p \alpha}$ and $F$ are decoupled, the Green function in Eq. (9) factorizes into $\mathcal{G}(\tau)=G_{0}(\tau) K(\tau)$, with $G_{0}(\tau)=\nu_{0} \pi T / \sin (\pi T \tau)$ being the free electron Green function and $K(\tau)=$ $\left\langle T_{\tau} F(\tau) F^{\dagger}(0)\right\rangle,[12]$.
Since the operator $F^{\dagger}(0)$ in $K(\tau)$ changes the value of $\hat{n}$ from zero to one at $t=0$, and $F(\tau)$ changes it back to zero at $t=\tau$, the correlator $K(\tau)$ can be rewritten as

$$
K(\tau)=\frac{Z(\tau)}{Z(0)}
$$

where $Z(\tau)$ is a functional integral over $\phi_{\alpha}$ 's in the presence of the time-dependent charge $n_{\tau}(t)=\theta(t) \theta(\tau-t)$. Introducing the charge and spin mode variables in the right contact $\phi_{c, s}(x)=\left[\phi_{\uparrow}(x) \pm \phi_{\downarrow}(x)\right] / \sqrt{2}$, we can write $Z(\tau)$ as

$$
Z(\tau)=\int D\left[\phi_{c}, \phi_{s}\right] \exp \left[-\mathcal{S}_{C}(\tau)-\mathcal{S}_{0, c}-\mathcal{S}_{0, s}-\mathcal{S}_{R}\right] .
$$

Here $\mathcal{S}_{0, c}+\mathcal{S}_{0, s}$ represents the free electron part of the action in the absence of backscattering in the QPC, $S_{C}$ denotes its charging part, and $S_{R}$ represents the backscattering in the QPC. These terms are given by

$$
\begin{gathered}
\mathcal{S}_{0, i}=\int_{0}^{\beta} d t \int d x \frac{v_{F}}{2 \pi}\left(\left[\nabla \phi_{i}\right]^{2}+\frac{\dot{\phi}_{i}^{2}}{v_{F}^{2}}\right), \quad i=c, s \\
\mathcal{S}_{C}(\tau)=\int_{0}^{\beta} d t E_{C}\left[n_{\tau}(t)+\frac{\sqrt{2}}{\pi} \phi_{c}(0, t)-N\right]^{2}, \\
\mathcal{S}_{R}=\int_{0}^{\beta} d t \frac{2 D}{\pi}|r| \cos \left[\sqrt{2} \phi_{c}(0, t)\right] \cos \left[\sqrt{2} \phi_{s}(0, t)\right] .
\end{gathered}
$$

At frequencies below $E_{C}$ the fluctuations of the charge mode, $\phi_{c}(0, t)$ are suppressed by the charging energy term (12b) and can be integrated out. Furthermore, we can evaluate the functional integral over $\phi_{c}$ by the saddle point approximation ignoring the backscattering term, Eq. (12c).

The action $\mathcal{S}^{\mathrm{sp}}(\tau)$ and the value of the charge mode $\phi_{c}^{\mathrm{sp}}(0, t)$ at the saddle point are found to be

$$
\begin{aligned}
\mathcal{S}^{\mathrm{sp}}(\tau) & =\mathcal{S}_{C}^{\mathrm{sp}}(\tau)+\mathcal{S}_{0, c}^{\mathrm{sp}}=\ln \frac{2 \gamma E_{C} \sin (\pi T \tau)}{\pi^{2} T}, \\
\sqrt{2} \phi_{c}^{\mathrm{sp}}(0, t) & =\pi\left[N-n_{\tau}(t)\right]+\mathcal{F}(t)+\mathcal{F}(\tau-t), \\
\mathcal{F}(t) & =\sum_{n=1}^{\infty} \frac{\sin (2 \pi n T t)}{n+\frac{E_{C}}{\pi^{2} T}} .
\end{aligned}
$$

In Eq. 13a we have assumed that $\tau \gg E_{C}^{-1}$, which is a good approximation since we only need $\tau=\beta / 2+i t$ in Eq. (8).

Averaging the backscattering term (12d) over the fluctuations of $\phi_{c}$ we obtain

$\tilde{\mathcal{S}}_{R, \tau}=\sqrt{\frac{8 \gamma E_{C} D}{\pi^{3}}}|r| \int_{0}^{\beta} d t \cos \left[\sqrt{2} \phi_{c}^{\mathrm{sp}}(0, t)\right] \cos \left[\sqrt{2} \phi_{s}(0, t)\right]$.

Since the charge modes can only be intergated out at frequencies below the charging energy, one has to assume that the energy cutoff in the above action is $D \sim E_{C}$. 
Equation (11) can now be written as

$$
\begin{aligned}
Z(\tau) & =\mathcal{N} e^{-\mathcal{S}^{\mathrm{sp}}(\tau)} Z_{s}(\tau), \\
Z_{s}(\tau) & =\int D\left[\phi_{s}\right] \exp \left(-\mathcal{S}_{0, s}-\tilde{\mathcal{S}}_{R, \tau}\right),
\end{aligned}
$$

where $\mathcal{N}$ is the $\tau$-independent factor which arises from the integration over the fluctuations about the saddle point and drops out of $K(\tau)$ in Eq. (10). The correlator $K(\tau)$ in Eq. (10) then factorizes into $K(\tau)=$ $K_{\Theta}(\tau) K_{F}(\tau)$, where

$$
K_{\Theta}(\tau)=e^{-\mathcal{S}^{\mathrm{sp}}(\tau)}=\frac{\pi^{2} T}{2 \gamma E_{C} \sin (\pi T \tau)},
$$

and $K_{F}(\tau)$ is the spin part of the correlator which can be expressed as

$$
K_{F}(\tau)=Z_{s}(\tau) / Z_{s}(0) .
$$

The effective action in Eq. (14b) can be re-fermionized following Refs. [11] 12]. The Hamiltonian in this representation has the form

$$
\begin{aligned}
& \hat{H}=i v_{F} \int \psi^{\dagger}(x) \nabla \psi(x) d x+\lambda(t) \eta\left[\psi(0)-\psi^{\dagger}(0)\right], \\
& \lambda(t)=\frac{2}{\pi} \sqrt{\gamma v_{F} E_{C}}|r| \cos \left[\sqrt{2} \phi_{c}^{\mathrm{sp}}(t)\right],
\end{aligned}
$$

where $\eta=\left(c+c^{\dagger}\right)$ is a Majorana fermion.

In the limit $T / E_{C} \rightarrow 0$ the functions $\mathcal{F}(t)$ in Eqs. (13b, 13c) tend to zero, and to the leading order in $T / E_{C}$ can be neglected 12]. Then the timedependent coefficient $\lambda(t)$ in Eq. (17) becomes $\lambda_{0}(t)=$ $\frac{2}{\pi} \sqrt{\gamma v_{F} E_{C}}|r|(-1)^{n_{\tau}(t)} \cos [\pi N]$. In this approximation 112 the odd component of the tunneling DoS in the dot vanishes, thus nullifying the thermopower. Therefore we expand $K_{F}(\tau)$ in Eq. (16) to first order in $\delta \lambda(t)=\lambda(t)-\lambda_{0}(t)$. In the fermion representation (17) we obtain for the linear in $\delta \lambda(t)$ correction to $K_{F}(\tau)$

$$
\begin{aligned}
\Delta K_{F}(\tau) & =\int_{0}^{\beta}(-1)^{n_{\tau}(t)} \delta \lambda(t) \Phi(\tau, t) d t, \\
\Phi(\tau, t) & =\left\langle T_{t} \eta(\tau) \eta(0) \eta(t)\left[\psi(0, t)-\psi^{\dagger}(0, t)\right]\right\rangle,
\end{aligned}
$$

where $\langle\ldots\rangle$ denotes the thermal average with the Hamiltonian (17) with $\lambda=\frac{2}{\pi} \sqrt{\gamma v_{F} E_{C}}|r| \cos [\pi N]$ independent of time $t$.

The average in Eq. (18b) can be evaluated with the aid of Wick theorem. It is not difficult to show that the thermopower is an odd function of $N$. We therefore need only to retain the odd in $N$ component $\Delta_{\text {odd }} K_{F}(\tau)$ of Eq. (18a). Evaluating the integral in Eq. (18a) with logarithmic accuracy in $E_{C} / \max \{T, \Gamma\}$ we find:

$$
\begin{aligned}
\Delta_{\text {odd }} K_{F}(\tau)= & -\frac{8}{E_{C}} \sqrt{\frac{\gamma \Gamma E_{C}}{\pi}}|r| \sin (\pi N) \ln \frac{E_{C}}{T+\Gamma} \\
& \times \int \frac{\xi d \xi}{\xi^{2}+\Gamma^{2}} \frac{e^{\xi|\tau|}}{e^{\beta \xi}+1} .
\end{aligned}
$$

The upper energy scale $E_{C}$ in the logarithmic factor originates from the above mentioned energy cutoff $D \sim E_{C}$ of the spin excitations. Using Eq. (19) we obtain our main result, Eq. (11).

In conclusion, we have presented a theory of the Coulomb blockade oscillations of the thermoelectric coefficient $G_{T}$ and the thermopower $S$ of quantum dots in the anisotropic nearly open regime in the limit where the single particle mean level spacing is negligible. Two distinct regimes can be identified: the one with $\Gamma \gg T$, and the one with $\Gamma \ll T$. In the former the thermopower is linear in temperature but is nearly independent of the reflection coefficient in the QPC and can be expressed in the form of Eq. (1) analogous to the Cutler-Mott formula [14]. In the latter, the thermopower is linear in the reflection coefficient $|r|^{2}$ but depends on the temperature only logarithmically.

We are grateful to B.L. Altshuler, C.M. Marcus, J.M. Martinis, L.W. Molenkamp and B.Z. Spivak for valuable discussions. It is our pleasure to acknowledge the warm hospitality of the Aspen Center for Physics, the ICTP, Trieste, and the Centre for Advanced Studies in Oslo where part of this work was performed. The authors are A.P. Sloan Research Fellows. A.A. is a Packard Research Fellow. This research was supported by the NSF Grants No. DMR-9984002 and DMR-9974435.

[1] A.V. Anisovich, B.L. Altshuler, A.G. Aronov, and A.Yu. Zyuzin, JETP Lett 45, 295 (1987).

[2] B.Z. Spivak and A.Yu. Zyuzin, Europhys.Lett. 8, 669 (1989).

[3] M. Nahum, T.M. Eiles, and J.M. Martinis, Appl. Phys. Lett. 653123 (1994).

[4] C.W.J. Beenakker and A.A.M. Staring, Phys. Rev. B46, 9667 (1992).

[5] A.A.M. Staring, L.W. Molenkamp, B.W. Alphenhaar, H. van Houten, O.J.A. Buyk, M.A.A. Mabesoone, C.W.J. Beenakker, and C.T. Foxon, Europhys. Lett. 22, 57 (1993).

[6] A.S. Dzurak, C.G. Smith, C.H.W. Barnes, M. Pepper, L. Martin-Moreno, C.T. Liang, D.A. Ritchie, and G.A.C. Jones, Phys. Rev. B55, R10197 (1997).

[7] M. Turek and K.A. Matveev, in preparation.

[8] S. Möller, H. Buhmann, S.F. Godijn, and L.W. Molenkamp, Phys. Rev. Lett. 81, 5197 (1998).

[9] I.L. Aleiner and L.I. Glazman, Phys. Rev. B, 57, 9608 (1998).

[10] K. Flensberg, Phys. Rev. B48, 11156 (1993); Physica (Amsterdam) 203B, 432 (1994).

[11] K.A. Matveev, Phys. Rev. B51, 1743 (1995).

[12] A. Furusaki and K.A. Matveev, Phys. Rev. Lett. 75, 709 (1995); Phys. Rev. B52, 16676 (1995).

[13] D.V. Averin and Yu.V. Nazarov, Phys. Rev. Lett. 65, 2446 (1990).

[14] M. Cutler and N.F. Mott, Phys. Rev. 181, 1336 (1969). 